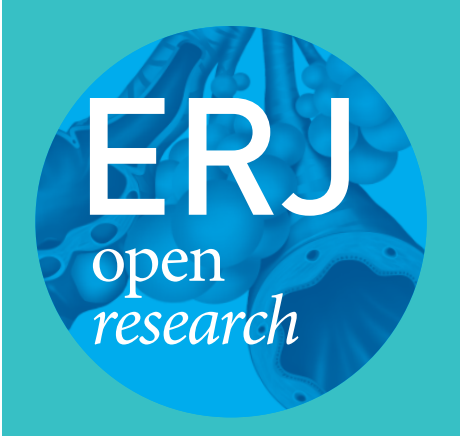

\title{
The role of chest radiography in the diagnosis of bacteriologically confirmed pulmonary tuberculosis in hospitalised Xpert MTB/RIF-negative patients
}

\author{
Wakjira Kebede ${ }^{1,2,3}$, Gemeda Abebe ${ }^{1,2}$, Esayas Kebede Gudina $\mathbb{1}^{4}$, Elias Kedir ${ }^{5}$, \\ Thuy Ngan Tran (i) $^{3}$ and Annelies Van Rie (10 ${ }^{3}$
}

ABSTRACT The role of chest radiography to diagnose active tuberculosis in symptomatic patients who have a negative Xpert MTB/RIF (Xpert) test result is unclear. This study aimed to assess the performance of chest radiography and the value of chest radiography findings for a prediction tool to identify cases of active pulmonary tuberculosis among symptomatic, Xpert-negative hospitalised patients.

Xpert-negative patients hospitalised between January and July 2019 at Jimma University Medical Center in Ethiopia were assessed by mycobacterial culture and chest radiography. Chest radiography was interpreted by a clinician for clinical decision making and by a radiologist for research purposes. Using bacteriological confirmation as the reference standard, the performance of chest radiography to diagnose active tuberculosis was assessed by the area under the receiver operating characteristic curve (AUC); predictors of active tuberculosis were identified using bivariate and multivariate logistic regression analyses.

Of 247 Xpert-negative patients, $38 \%$ and $40 \%$ were classified as suggestive of tuberculosis by clinician and radiologist, respectively. Of the 39 (15.8\%) bacteriologically confirmed cases, $69 \%$ and $79 \%$ were classified as having chest radiography findings suggestive of tuberculosis by clinician or radiologist, respectively. While there was a strong association between bacteriologically confirmed tuberculosis and chest radiography classified by clinician as suggestive of tuberculosis (adjusted OR 2.7, 95\% CI 1.2-6.6), chest radiography with signs typical of tuberculosis (adjusted OR 5.3, 95\% CI 2.1-14.4) or compatible with tuberculosis (adjusted OR 5.1, 95\% CI 1.3-20.0), the positive predictive value of the chest radiography was low $(27 \%$ and $34 \%$ for classification by clinician and radiologist, respectively). The addition of chest radiography findings by clinician or radiologist to clinical characteristics did not improve the performance of the prediction tool, with similar risk classification distribution, AUCs and negative and positive prediction values.

Despite the strong association between chest radiography findings and active tuberculosis among hospitalised Xpert negative individuals, chest radiography findings did not improve the performance of a risk prediction tool based solely on clinical symptoms. Countries with a high tuberculosis/HIV burden should urgently replace Xpert by the more sensitive Xpert Ultra assay to improve the diagnosis of active tuberculosis.

@ERSpublications

In hospitalised people with symptoms of TB and a negative Xpert assay, knowledge of chest radiography findings does not improve the ability of clinicians to predict the presence of active TB beyond what is possible based solely on clinical characteristics https://bit.ly/30s $72 \mathrm{mX}$

Cite this article as: Kebede W, Abebe G, Gudina EK, et al. The role of chest radiography in the diagnosis of bacteriologically confirmed pulmonary tuberculosis in hospitalised Xpert MTB/RIFnegative patients. ERJ Open Res 2021; 7: 00708-2020 [https://doi.org/10.1183/23120541.00708-2020].

This article has supplementary material available from openres.ersjournals.com.

Received: 29 Sept 2020 | Accepted: 13 Nov 2020

Copyright $\odot$ ERS 2021. This article is open access and distributed under the terms of the Creative Commons Attribution Non-Commercial Licence 4.0. 


\section{Introduction}

Tuberculosis remains a major public health problem, with an estimated 10 million new active tuberculosis cases and 1.3 million deaths in 2019 [1]. Of the 10 million people diagnosed globally with active tuberculosis in 2019, 87\% occurred in 30 high tuberculosis burden countries. Ethiopia has the 10th highest burden of tuberculosis with 114233 cases corresponding to an estimated incidence rate of 151 tuberculosis cases per 100000 people [1,2].

The World Health Organization (WHO) strongly recommends the use of Xpert MTB/RIF (Xpert) assay (and since 2017 the Xpert Ultra assay) as the initial diagnostic test for all adults and children with signs and symptoms of tuberculosis [3]. WHO also conditionally recommends that Xpert (or Xpert Ultra) be used for further testing of smear-negative specimens in resource-constrained countries where multidrugresistant tuberculosis or HIV are of lesser concern [4]. Despite the progress made in biological diagnosis of active tuberculosis following the implementation of the Xpert assay, an estimated one in three tuberculosis cases globally remain undiagnosed.

In the pre-Xpert era, WHO viewed chest radiography as an important tool to diagnose pulmonary tuberculosis (PTB) when the diagnosis cannot be confirmed bacteriologically [5]. Several studies indeed demonstrated that a chest radiography is highly sensitive and can enhance the diagnosis of tuberculosis, especially in severely ill and HIV-positive patients, albeit with low specificity $[6,7]$ due to the presence of similar chest radiography findings in people with respiratory infections other than tuberculosis [8]. Furthermore, important variation between clinicians and radiologists in chest radiography interpretation can affect its specificity $[9,10]$.

In 2007, WHO recommended that chest radiography should be used in people presenting with symptoms of tuberculosis after an initial negative bacteriological test, a course of broad-spectrum antibiotics and a second negative round of bacteriological testing [5]. In 2008, WHO also recommended the use of chest radiography directly after an initial negative smear microscopy test [11].

In the Xpert era, there has been a lot of interest in the use of chest radiography as a triaging tool to limit the use of the relatively expensive Xpert assays. Little attention has been paid to the role of chest radiography in people with presumptive tuberculosis who have a negative initial Xpert assay result. This study aimed to assess added value of chest radiography (read by clinician or radiologist) to identify cases of active tuberculosis among people admitted to a tertiary hospital with symptoms of tuberculosis who had a negative Xpert result.

\section{Methods}

Study setting

The study was conducted from January to July 2019 at Jimma University Medical Center (JUMC) referral hospital that provides inpatient and outpatient services to the over 15 million people living in southwest Ethiopia [12]. At the JUMC, the Xpert test is used as the initial diagnostic in people presenting with symptoms or signs of PTB. When the Xpert assay is negative for Mycobacterium tuberculosis, the clinician can request chest radiography, paid by the individual seeking care, for clinical decision-making [13].

\section{Study population and data collection}

This analysis is a secondary analysis of a cohort study that aimed to assess the role of empiric treatment in hospitalised adult (age $\geqslant 18$ years) patients with symptoms of PTB and negative Xpert result [14]. In the parent study, 125 patients treated for active tuberculosis and 125 patients in whom the physician decided not to treat for tuberculosis were enrolled. All patients were assessed by chest radiography and sputum mycobacterial culture prior to tuberculosis treatment initiation. Chest radiography was not performed at the end of treatment.

Mycobacterial culture was used as the gold standard for diagnosis of tuberculosis. About $0.5 \mathrm{~mL}$ of the pellet of a sputum sample subjected to $\mathrm{N}$-acetyl-L-cysteine-sodium hydroxide was inoculated on the BACTEC MGIT 960 system and incubated for up to 42 days; another $0.5 \mathrm{~mL}$ of the pellet was inoculated on solid Lowenstein-Jensen media at $37^{\circ} \mathrm{C}$ for 56 days. M. tuberculosis growth was confirmed by the Ziehl-Neelsen stain and Capilla test. Cultures with non-acid-fast bacilli growth on blood agar were

Affiliations: ${ }^{1}$ Mycobacteriology Research Center, Jimma University, Jimma, Ethiopia. ${ }^{2}$ School of Medical Laboratory Science, Institute of Health, Jimma University, Jimma, Ethiopia. ${ }^{3}$ Dept of Family Medicine and Population Health (FAMPOP), Faculty of Medicine and Health Sciences, University of Antwerp, Antwerp, Belgium. ${ }^{4}$ Dept of Internal Medicine, Jimma University Medical Center, Jimma University, Jimma, Ethiopia.

${ }^{5}$ Dept of Radiology, Jimma University Medical Center, Jimma University, Jimma, Ethiopia.

Correspondence: Wakjira Kebede, 378, Jimma University, Ethiopia. E-mail: wakjirakebededyahoo.com 
re-decontaminated and re-incubated [15]. In case of contamination of both solid and liquid culture, a single Xpert Ultra test was performed on a stored sputum sample according to manufacturer's guideline or explained in literature $[4,16]$. Participants were classified as cases of bacteriologically confirmed tuberculosis when $M$. tuberculosis complex grew on liquid and/or solid culture media or when $M$. tuberculosis was detected on Xpert Ultra.

Relevant socio-demographic and clinical data were collected via a pre-tested structured questionnaire and retrieved from the participants' medical records. Data collected were age, sex, educational level, marital status, body mass index (BMI), weight loss, chest pain, loss of appetite, history of tuberculosis treatment, presence and duration of cough, fever, night sweats, and shortness of breath, HIV status and CD4 count.

The classification of chest radiography by a team of clinicians as "suggestive of tuberculosis" or "not suggestive of tuberculosis" as part of routine clinical care was retrieved from the medical records. For research purpose, a senior radiologist who was blinded to chest radiography reading by the clinician and the patient's clinical and laboratory data, classified chest radiography as normal, compatible with tuberculosis (enlarged hilar nodes, pneumonic lesion, atelectasis, mass lesion, or miliary abnormalities) or typical for tuberculosis (nodular, alveolar, or interstitial infiltrates affecting the zones above the clavicles or upper zones of the lungs) using a standardised classification system [17].

\section{Data analysis}

Baseline characteristics of study participants and chest radiography abnormalities are reported as frequencies and proportions. Kappa score was calculated to assess the inter-reader agreement of chest radiography classification between clinicians and senior radiologist. For this analysis, the radiologist's readings of "typical of tuberculosis" and "compatible with tuberculosis" were combined into a single "suggestive of tuberculosis" category to create a binary classification of "suggestive of tuberculosis" or "not suggestive of tuberculosis". Using bacteriologically confirmed tuberculosis as the gold standard, sensitivity, specificity, negative predictive value (NPV) and positive predictive value (PPV) were estimated for chest radiography classification by clinician and radiologist.

Bivariate logistic regression models were used to determine the association between clinical variables (sex, symptoms of tuberculosis, BMI and HIV infection), radiological findings (by clinician or radiologist), and bacteriologically confirmed PTB. Three multivariate models were built to assess the association between bacteriologically confirmed tuberculosis and clinical characteristics (model 1), clinical characteristics and chest radiography interpretation by clinician (model 2) and clinical characteristics and chest radiography interpretation by radiologist (model 3). Variables included in multivariate logistic regression model were those with a p-value $<0.2$ in bivariate analysis. Generalised variance-inflation factor (GVIF) was estimated to check multicollinearity among variables in multivariate models [18]. Backward stepwise model reduction was performed using the likelihood ratio test with a p-value cut-point of 0.1 .

The predictive accuracy of the final models was assessed by calibration and discrimination parameters. Model discrimination was estimated by the area under a receiver operating characteristic curve (AUC) with a range from 0.5 indicating no discrimination to 1.0 indicating perfect discrimination. Model calibration was assessed visually with a calibration plot and by comparing the predicted and observed probabilities using the Hosmer-Lemeshow test ( $p>0.05$ indicates good fit). The models were internally validated using a non-parametric bootstrap approach with 1000 random bootstrap samples. The extent of performance over-optimism when the models are applied to new patients in a similar population was measured by the average AUC difference between the bootstrap samples and the original full sample.

To assess the value of chest radiography results in addition to clinical characteristics, the three prediction models were transformed to simplified risk scores. For each model, the coefficient of each variable was divided by the smallest coefficient and rounded to the nearest integer to give the weight for each variable in the simplified risk score. For each of the three models, a risk score was calculated for each individual participant and each patient's risk of bacteriologically confirmed tuberculosis was classified into one of four risk categories (very low, low, moderate and high risk).

Finally, using different cut-offs, the diagnostic performance of chest radiography in diagnosing active PTB was assessed by calculating sensitivity, specificity, positive and negative predictive values. All data analyses were performed using the R Statistical software version 3.6.1.

\section{Ethical considerations}

The study was approved by the institutional review board (IRB) of Jimma University Institute of Health (Ref. no. IHRPGD 397/2018). Written informed consent was obtained from all study participants. Patients with bacteriologically confirmed tuberculosis on study samples were started on tuberculosis treatment. 


\section{Results}

\section{Baseline characteristics}

Of 250 patients enrolled in the parent study, 247 patients with data on both chest radiography and bacteriological evaluation were included in this analysis (figure 1). Baseline characteristics of the study population are shown in (table 1). Median age was 39 years, about half (55.5\%) were male, most were married (61.5\%), had a primary school education (66.8\%) and were rural residents $(57.9 \%)$. One in three (34.0\%) were underweight (BMI $\leqslant 18.5 \mathrm{~kg} \cdot \mathrm{m}^{-2}$ ) and among the $240(97 \%)$ patients tested, $52(21.7 \%)$ were HIV positive. All participants self-reported one or more tuberculosis symptoms, including weight loss $(55.0 \%)$, chest pain $(66.8 \%)$, prolonged cough $(\geqslant 2$ weeks) $(65.6 \%)$, fever $(45.3 \%)$, night sweats $(48.2 \%)$ and shortness of breath (50.6\%). Few (18.2\%) self-reported a history of tuberculosis treatment.

\section{Chest radiography findings}

According to the clinician who read the chest radiography as part of routine clinical care, 99 (40.1\%) participants had chest radiography suggestive of tuberculosis and 148 (59.9\%) had chest radiography not suggestive of tuberculosis. According to the radiologist, 73 (29.6\%) patients had typical chest radiography findings for tuberculosis (31 cavitary lesion, 26 pleural effusion and 16 consolidation), 20 (8.1\%) had chest radiography compatible with tuberculosis (14 miliary disease, four fibrosis and two hilar adenopathy) and $154(62.3 \%)$ had chest radiography not suggestive of active tuberculosis (table 2). The agreement between the classification of chesty radiography reading by clinician (suggestive of tuberculosis versus not suggestive of tuberculosis) and radiologist (typical or compatible with tuberculosis versus not suggestive of tuberculosis) was good $(87.1 \%$ agreement; $\kappa$ score $=0.728, \mathrm{p}<0.001)($ table 3$)$.

Of the 39 participants with bacteriologically confirmed PTB, the radiologist classified 23 (59.0\%) patients' chest radiography as having typical chest radiography findings of tuberculosis, eight (20.5\%) as having signs compatible with tuberculosis and eight (20.5\%) as having no abnormalities suggestive of tuberculosis (table 2). All eight patients with normal chest radiography but bacteriologically confirmed PTB were HIV positive. The clinician classified the chest radiography of $27(69.2 \%)$ of the 39 patients with bacteriologically confirmed as suggestive of tuberculosis, and $12(30.8 \%)$ as not suggestive of tuberculosis. The sensitivity of the clinician's chest radiography reading to identify a case of bacteriologically confirmed tuberculosis was 69\% (95\% CI 63-90), specificity 65\% (95\% CI 58-71), PPV 27\% (95\% CI 22-33) and NPV 92\% (95\% CI 88-95). The sensitivity of the radiologist's reading to identify cases of bacteriologically confirmed tuberculosis was 79.5\% (95\% CI 64-90), specificity 70\% (95\% CI 64-76), PPV 34\% (95\% CI 28-39) and NPV 95\% (95\% CI 90-97).

250 hospitalised presumptive Xpert-negative patients identified

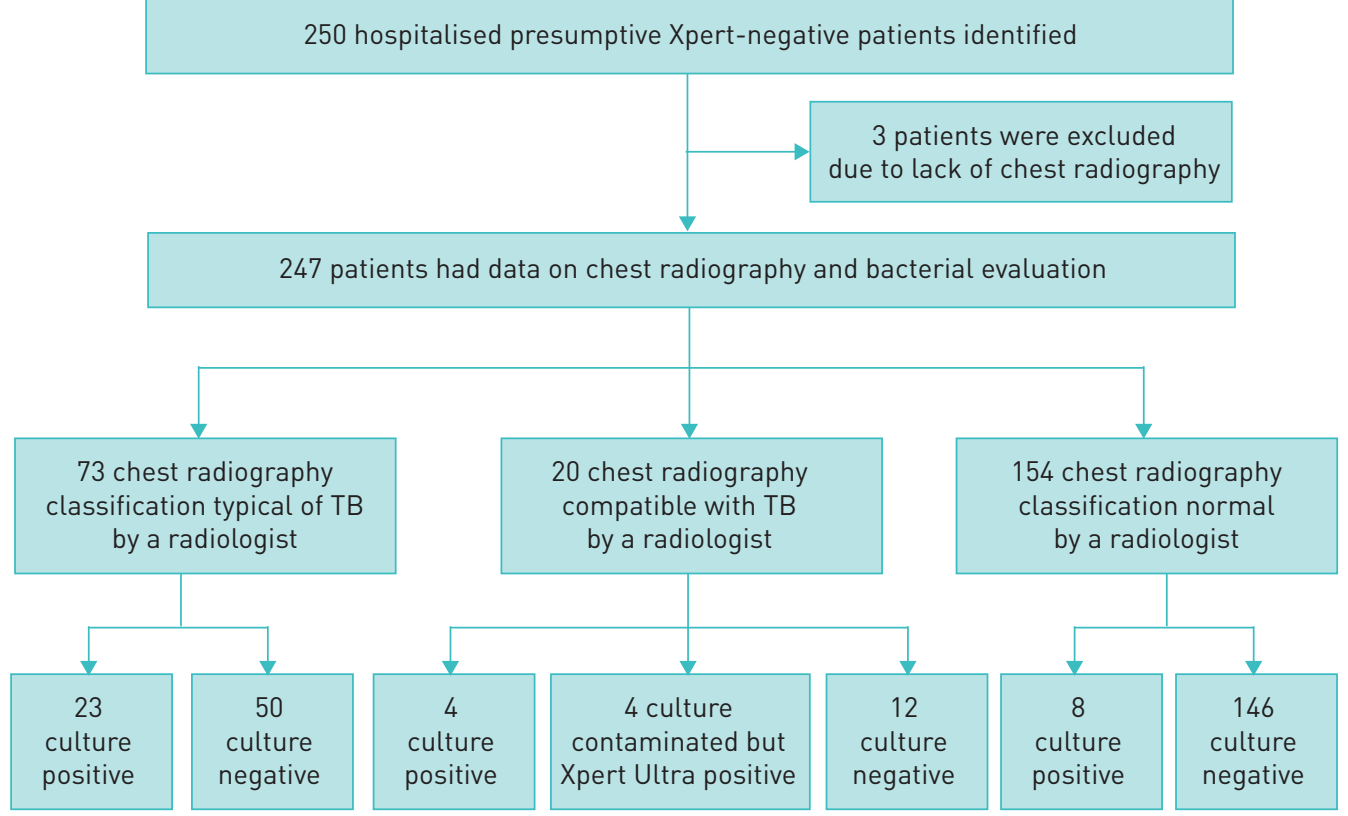

FIGURE 1 Flow chart of enrolment, chest radiography classification by radiologist and results of laboratory tests among 247 Xpert-negative adults hospitalised at Jimma University Medical Center, Ethiopia. TB: tuberculosis. 
Ability of chest radiography to diagnose bacteriologically confirmed tuberculosis among patients with a negative Xpert result

Eight clinical variables and chest radiography reading (by clinician or radiologist) were associated with bacteriologically confirmed PTB in bivariate logistic regression (table 4). All variables in the initial multivariate models showed GVIFs <2 (1.06-1.46), indicating absence of multicollinearity. After backward stepwise model reduction, model 1 (clinical parameters only) comprised five variables: age, loss of appetite, BMI, prolonged cough and prolonged shortness of breath. In model 2 (clinical parameters and clinician chest radiography reading), four clinical variables (age, loss of appetite, BMI and prolonged cough) and chest radiography reading were included. Compared with patients with chest radiography not suggestive of tuberculosis, the odds of bacteriologically confirmed tuberculosis was 2.7 (95\% CI 1.2-6.6) times higher for patients with chest radiography suggestive of tuberculosis. In model 3 (clinical parameters and chest radiography reading by radiologist), four clinical variables (age, BMI, loss of appetite and prolonged cough) and chest radiography reading were included. Compared with patients with chest radiography not suggestive of tuberculosis, the odds of bacteriologically confirmed tuberculosis was 5.3 (95\% CI 2.1-14.4) times higher for patients with typical chest radiography signs of tuberculosis and 5.1 (95\% CI 1.3-20.0) times higher for patients with chest radiography signs compatible with tuberculosis.

TABLE 1 Characteristics of 247 Xpert-negative presumptive tuberculosis (TB) cases

hospitalised at Jimma University Medical Center, southwest Ethiopia

\begin{tabular}{|c|c|}
\hline Characteristics & Number \\
\hline \multicolumn{2}{|l|}{ Age } \\
\hline $18-40$ years & $141(57.1)$ \\
\hline$\geqslant 41$ years & 106 (42.9) \\
\hline \multicolumn{2}{|l|}{ Sex } \\
\hline Female & 110 (44.5) \\
\hline Male & $137(55.5)$ \\
\hline \multicolumn{2}{|l|}{ Marital status } \\
\hline Single & 58 (23.5) \\
\hline Married & 152 (61.5) \\
\hline Widowed or divorced & $37(15.0)$ \\
\hline \multicolumn{2}{|l|}{ Educational level } \\
\hline Illiterate & $114(46.2)$ \\
\hline Primary & $51(20.6)$ \\
\hline Secondary and above & 82 (33.2) \\
\hline \multicolumn{2}{|l|}{ Residence } \\
\hline Urban & $104(42.1)$ \\
\hline Rural & 143 (57.9) \\
\hline \multicolumn{2}{|l|}{ Body mass index } \\
\hline$>18.5 \mathrm{~kg} \cdot \mathrm{m}^{-2}$ & $163(66.0)$ \\
\hline$\leqslant 18.5 \mathrm{~kg} \cdot \mathrm{m}^{-2}$ & 84 (34.0) \\
\hline \multicolumn{2}{|l|}{ Presence of co-morbidities } \\
\hline Diabetes mellitus & 7 (3) \\
\hline COPD & $18(7)$ \\
\hline \multicolumn{2}{|l|}{ HIV status ${ }^{\#}$} \\
\hline Negative & 188 (78.3) \\
\hline Positive & $52(21.7)$ \\
\hline \multicolumn{2}{|l|}{ CD4 count ( $n=52)$} \\
\hline$\leqslant 200$ cells $\mu \mathrm{L}^{-1}$ & $25(48.1)$ \\
\hline$>200$ cells $\cdot \mu \mathrm{L}^{-1}$ & 27 (51.9) \\
\hline History of TB treatment & 45 (18.2) \\
\hline Severely ill & $17(6.9)$ \\
\hline Loss of appetite & $199(80.6)$ \\
\hline Weight loss & $136(55.0)$ \\
\hline Chest pain & 165 (66.8) \\
\hline Cough of $\geqslant 2$ weeks & $162(65.6)$ \\
\hline Fever of $\geqslant 2$ weeks & 112 (45.3) \\
\hline Night sweat of $\geqslant 2$ weeks & $119(48.2)$ \\
\hline Shortness of breath of $\geqslant 2$ weeks & $125(50.6)$ \\
\hline
\end{tabular}


TABLE 2 Chest radiography classification by clinicians and radiologist, stratified by bacteriological tuberculosis (TB) confirmation status in 247 Xpert-negative patients hospitalised at Jimma University Medical Center, Ethiopia

\begin{tabular}{|c|c|c|c|}
\hline Chest radiography findings & All & $\begin{array}{l}\text { Bacteriologically } \\
\text { confirmed for TB }\end{array}$ & $\begin{array}{l}\text { Not bacteriologically } \\
\text { confirmed for TB }\end{array}$ \\
\hline Patients n & 247 & 39 & 208 \\
\hline \multicolumn{4}{|c|}{ Chest radiography classified by clinicians } \\
\hline Not suggestive of TB & $148(59.9)$ & $12(30.8)$ & $136(65.4)$ \\
\hline Suggestive of TB & $99(40.1)$ & $27(69.2)$ & $72(34.6)$ \\
\hline \multicolumn{4}{|c|}{ Chest radiography classified by a radiologist } \\
\hline Not suggestive of TB & 154 (62.3) & $8(20.5)$ & $146(70.2)$ \\
\hline Typical of TB & $73(29.6)$ & $23(59.0)$ & $50(24.0)$ \\
\hline Cavitary lesion & $31(12.6)$ & $11(28.2)$ & $20(9.6)$ \\
\hline Pleural effusion & $26(10.5)$ & $6(15.4)$ & $20(9.6)$ \\
\hline Consolidation & $16(6.5)$ & $6(15.4)$ & $10(4.8)$ \\
\hline Compatible with TB & $20(8.1)$ & $8(20.5)$ & $12(5.8)$ \\
\hline Miliary disease & $14(5.7)$ & $6(15.4)$ & 8 (3.8) \\
\hline Fibrosis & $4(1.6)$ & $1(2.6)$ & $3(1.4)$ \\
\hline Hilary adenopathy & $2(0.8)$ & $1(2.6)$ & $1(0.5)$ \\
\hline
\end{tabular}

The contribution (weighted points) of the variables to the risk scores for the different models was estimated and a risk score calculated for each individual patient using clinical parameters only (model 1), clinical parameters plus chest radiography reading by clinicians (model 2), and clinical parameters plus chest radiography reading by radiologist (model 3) (table 5). The distribution of risk scores and classification in four risk categories for bacteriologically confirmed tuberculosis (very low, low, moderate and high risk) for each of the three models is presented in table 6 . For all three models, $0 \%$ of patients with a very low risk, and about 70\% of patients with high risk (74\% for model 1, 67\% for model 2 and $70 \%$ for model 3) had bacteriologically confirmed tuberculosis.

The calibration plots of models 1,2 and 3 showed good calibration with predicted probabilities reasonably similar to the observed probabilities across the distribution of risk (figure S1). The AUCs of the final multivariate models 1, 2 and 3 were 0.842 (95\% CI 0.77-0.90), 0.845 (95\% CI 0.77-0.91) and 0.857 (95\% CI 0.78-0.92), respectively (figure S2). After bootstrapping, the estimated optimism in the three models' AUC was shown to be very low (mean range 0.0227-0.0239) and the calibration plot showed that predicted probabilities and observed probabilities were almost similar (figure S3).

The simplified risk prediction tools built based on the three models yielded very similar AUCs to that of the final multivariable models, with little difference between the three models: 0.835 (95\% CI $0.77-0.90$ ) for risk tool $1,0.845$ (95\% CI $0.78-0.91)$ for risk tool 2 and 0.856 (95\% CI 0.78-0.92) for risk tool 3 (figure S4).

TABLE 3 The inter-reader agreement between the clinicians and a radiologist on the chest radiography classification

\begin{tabular}{|c|c|c|c|c|c|}
\hline & \multicolumn{3}{|c|}{ Radiograph read by a radiologist ${ }^{\#}$} & \multirow[t]{2}{*}{ Kappa (к) } & \multirow[t]{2}{*}{ p-value } \\
\hline & $\begin{array}{l}\text { Typical of TB or } \\
\text { compatible with TB }\end{array}$ & $\begin{array}{c}\text { Not suggestive } \\
\text { of TB }\end{array}$ & Total & & \\
\hline \multicolumn{6}{|l|}{$\begin{array}{l}\text { Radiograph read by } \\
\text { clinicians }\end{array}$} \\
\hline Suggestive of TB & $80(86.0)$ & 19 (12.3) & $99(40.1)$ & 0.728 & $<0.001$ \\
\hline Not suggestive of TB & $13(14.0)$ & $135(87.7)$ & $148(59.9)$ & & \\
\hline Total & 93 (37.7) & $154(62.3)$ & $247(100)$ & & \\
\hline \multicolumn{6}{|c|}{$\begin{array}{l}\text { Data are presented as } n(\%) \text {, unless otherwise stated. TB: tuberculosis. }{ }^{\#} \text { : radiologist classified chest } \\
\text { radiography for research purpose, using the standardised methodology to classify each chest radiograph } \\
\text { [17]: П?: clinicians classified the chest radiography as part of routine care, for patient management. }\end{array}$} \\
\hline
\end{tabular}


TABLE 4 Association between potential predictor variables and bacteriologically confirmed tuberculosis (TB) among 247 symptomatic hospitalised patients with negative Xpert MTB/RIF result

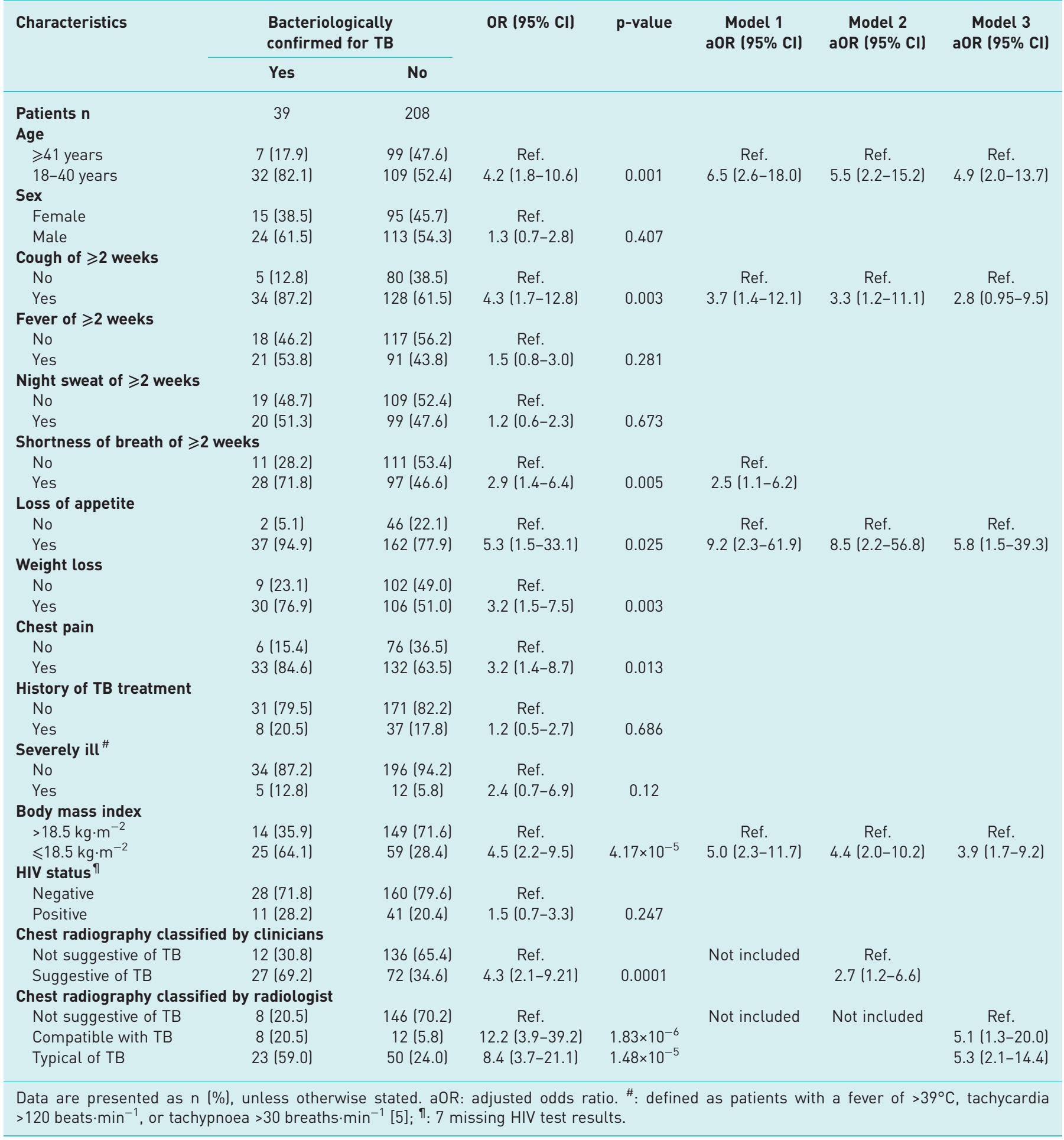

The sensitivity, specificity and predictive values for specific risk score cut-offs are presented in table 7 . If, for model 1 (clinical parameters only), a cut-off of 8 (high risk versus other categories) is chosen as the threshold for empiric treatment decision making, the NPV and specificity for confirmed PTB were high ( $89 \%$ and $98 \%$, respectively) but the sensitivity and PPV were relatively low ( $36 \%$ and $74 \%$, respectively). Adding chest radiography reading by clinician or radiologist did not improve the performance of the risk prediction tool, with similarly low sensitivity and PPV. 
TABLE 5 Weighted points assigned to each of the independent variables significantly associated with bacteriologically confirmed tuberculosis (TB) in multivariate analysis

\begin{tabular}{|c|c|c|c|c|c|c|}
\hline \multirow[t]{2}{*}{ Characteristics } & \multicolumn{2}{|c|}{ Model 1} & \multicolumn{2}{|c|}{ Model 2} & \multicolumn{2}{|c|}{ Model 3} \\
\hline & $\begin{array}{c}\text { Regression } \\
\text { coefficient }\end{array}$ & $\begin{array}{l}\text { Contribution to } \\
\text { risk score }\end{array}$ & $\begin{array}{l}\text { Regression } \\
\text { coefficient }\end{array}$ & $\begin{array}{l}\text { Contribution to } \\
\text { risk score }\end{array}$ & $\begin{array}{l}\text { Regression } \\
\text { coefficient }\end{array}$ & $\begin{array}{l}\text { Contribution to } \\
\text { risk score }\end{array}$ \\
\hline Age $18-40$ years & 1.8684 & 2 & 1.7057 & 2 & 1.5845 & 2 \\
\hline Cough of $\geqslant 2$ weeks & 1.3070 & 1 & 1.2016 & 1 & 1.0239 & 1 \\
\hline Shortness of breath of $\geqslant 2$ weeks & 0.9208 & 1 & & & & \\
\hline $\begin{array}{c}\text { Chest radiography classified as } \\
\text { suggestive of TB by clinician }\end{array}$ & Not included & Not included & 1.0026 & 1 & Not included & Not included \\
\hline $\begin{array}{l}\text { Chest radiography classified as } \\
\text { typical of TB by radiologist }\end{array}$ & Not included & Not included & Not included & Not included & 1.6275 & 2 \\
\hline $\begin{array}{l}\text { Chest radiography classified as } \\
\text { compatible with TB by } \\
\text { radiologist }\end{array}$ & Not included & Not included & Not included & Not included & 1.6679 & 2 \\
\hline
\end{tabular}

Model 1: risk score 2 if age (18-40 years) +1 if cough of $\geqslant 2$ weeks +1 if shortness of breath of $\geqslant 2$ weeks +2 if body mass index $\leqslant 18.5 \mathrm{~kg} \cdot \mathrm{m}^{-2}+2$ if loss of appetite. Model 2: risk score 2 if age (18-40 years) +1 if cough of $\geqslant 2$ weeks +1 if body mass index $\leqslant 18.5 \mathrm{~kg} \cdot \mathrm{m}^{-2}+2$ if loss of appetite +1 if chest radiography classification suggestive of TB by clinicians. Model 3: risk score 2 if age (18-40 years) +1 if cough of $\geqslant 2$ weeks +1 if body mass index $\leqslant 18.5 \mathrm{~kg} \cdot \mathrm{m}^{-2}+2$ if loss of appetite +2 if chest radiography classification typical/compatible of TB by radiologist. Shortness of breath of $\geqslant 2$ weeks was excluded due to lack of association in models 2 and 3.

TABLE 6 Distribution of risk scores and risk category among all 247 symptomatic hospitalised patients with negative Xpert result and among those with bacteriologically confirmed tuberculosis

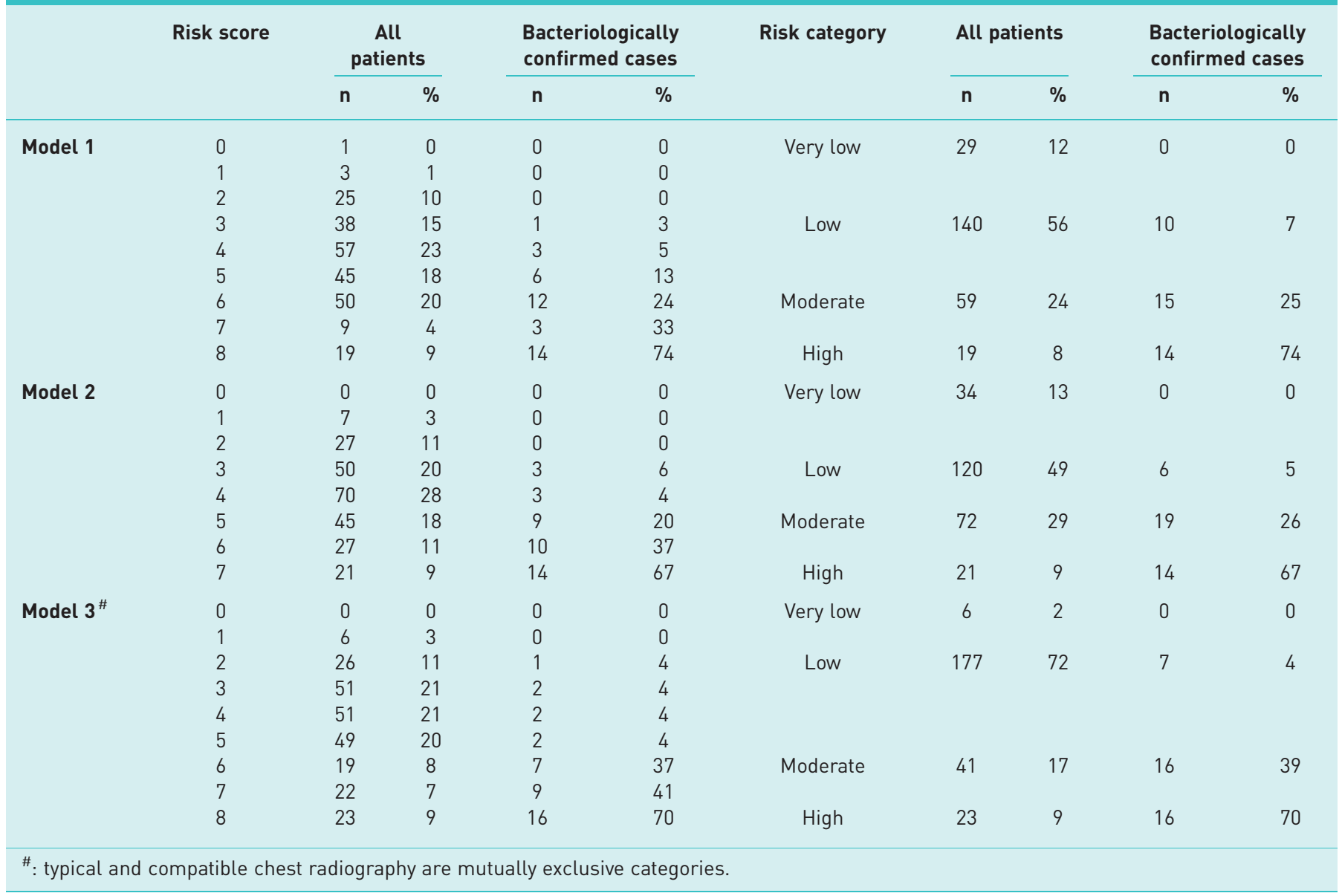


TABLE 7 The performance of chest radiography to diagnose active pulmonary tuberculosis among 247 Xpert-negative patients using bacteriological confirmation as a reference standard

\section{$\mathrm{n}[\%]$}

Sensitivity $(95 \% \mathrm{CI})$

\section{Specificity $(95 \% \mathrm{CI})$}

PPV $(95 \% \mathrm{CI})$

NPV $(95 \% \mathrm{Cl})$

\begin{tabular}{|c|c|c|c|c|c|}
\hline \multicolumn{6}{|c|}{ Clinical parameters only ${ }^{\#}$} \\
\hline Cut-off score $\geqslant 3$ & 218 (88.3) & 100 (91-100) & $14(10-19)$ & $18(13-24)$ & $100(88-100)$ \\
\hline Cut-off score $\geqslant 6$ & $78(31.6)$ & $74(58-87)$ & $76(70-82)$ & $37(26-49)$ & $94(89-97)$ \\
\hline Cut-off score 8 & $19(7.8)$ & $36(21-53)$ & 98 (94-99) & $74(49-91)$ & 89 (84-93) \\
\hline Cut-off score $\geqslant 3$ & $213(86.2)$ & $100(91-100)$ & $16(12-22)$ & $18(13-24)$ & 100 (90-100) \\
\hline Cut-off score $\geqslant 5$ & 93 (37.7) & 85 (69-94) & $71(64-77)$ & $35(26-46)$ & 96 (92-99) \\
\hline Cut-off score 7 & $21(8.5)$ & $36(21-53)$ & 97 (93-99) & $67(43-85)$ & 89 (84-93) \\
\hline Cut-off score $\geqslant 6$ & $64(26.0)$ & $82(66-92)$ & 85 (79-89) & 50 (37-63) & 96 (92-98) \\
\hline Cut-off score 8 & 23 (9.3) & $41(26-58)$ & 97 (93-99) & 70 (47-87) & 90 (85-93) \\
\hline
\end{tabular}

\section{Clinical parameters only}

$76(70-82)$

$37(26-49)$

94 (89-97)

by clinicians

$3(86.2)$

$93(37.7)$

85 (69-94)

71 (64-77)

$35(26-46)$

96 (92-99)

cut-off score 7

radiologis

$64(26.0)$

$82(66-92)$

$85(79-89)$

$16(12-21)$

$50(37-63)$

$96(92-98)$

90 (85-93)

\section{Discussion}

Among hospitalised patients with a negative Xpert result, we found that $15.8 \%$ had bacteriologically confirmed tuberculosis, about $40 \%$ had radiological signs of tuberculosis and about $70 \%$ of patients with bacteriologically confirmed tuberculosis had chest radiography findings suggestive of PTB. Despite the strong association between signs of tuberculosis on chest radiography and bacteriological confirmed tuberculosis, the addition of chest radiography findings to selected clinical symptoms did not improve the performance of a risk prediction tool. Availability of chest radiography findings for Xpert-negative patients assessed for tuberculosis at a tertiary care hospital in a high-tuberculosis burden country is thus unlikely to substantially improve the performance of empiric tuberculosis treatment decision-making based solely on clinical patient characteristics, which is common practice in many high-tuberculosis burden low-resource countries.

In the pre-Xpert era, WHO recommended the use of chest radiography in severely ill or smear-negative patients, especially in resource-constrained settings $[19,20]$. This recommendation was based on the high sensitivity of chest radiography findings for diagnosis of bacteriologically confirmed tuberculosis among smear-negative patients, with observed sensitivity ranging between 53.3\% (95\% CI 28-79) and 77\% (95\% CI 63-87) in prior studies [21-23]. In the Xpert era, chest radiography has been promoted in triaging algorithms to reduce the need for Xpert assays [24] and to improve case detection in tuberculosis prevalence surveys [7, 25], but not yet as a follow up test in Xpert negative patients. We observed sensitivity of chest radiography to diagnose bacteriologically confirmed tuberculosis fell similar to that in smear-negative individuals, with sensitivity estimates of $69 \%$ when chest radiography was read by clinicians and $79.5 \%$ when read by a radiologist, no other studies have reported on the sensitivity of chest radiography among Xpert-negative patients.

Because empiric treatment did not impact 6-month survival in this population of hospitalised patients with Xpert-negative PTB [14], we assessed if the addition of chest radiography findings to clinical characteristics in risk prediction models could improve the identification of patients with bacteriologically confirmed tuberculosis. Prediction models using clinical variables only (loss of appetite, BMI, prolonged cough, shortness of breath and age) or a combination of clinical characteristics and chest radiography findings (as defined by clinician or radiologist) were developed, internally validated and used to categorise patients into four distinctive risk groups ranging from low to very high risk of bacteriologically confirmed tuberculosis. We showed that the performance of the prediction tools containing chest radiography information was not superior in AUC or risk category distribution compared with the prediction tools containing only information on clinical patient characteristics. These results suggest that the inclusion of chest radiography images read by clinicians or radiologist is unlikely to improve the performance of empiric tuberculosis treatment in these populations. Whether the use of computer-aided reading to classify chest radiography findings could increase the role of chest radiography in Xpert-negative individuals should be explored in future studies.

To improve diagnostic accuracy and early treatment of all tuberculosis cases, greater emphasis should be placed on the use of Xpert Ultra, the most sensitive bacteriological assay. For example, even though Xpert 
Ultra was already recommended by WHO in 2017, the Global Fund 2020 procurement for Ethiopia consisted of $80 \%$ first-generation Xpert assays and only 20\% Xpert Ultra assays [26, 27]. Where procurement of Xpert Ultra is not possible, the clinical risk prediction tool suggests that targeting empiric treatment to those at moderate or high risk of bacteriological tuberculosis may be an effective strategy, but this would need to be confirmed in prospective studies [3].

Our study had some limitations. First, the subjects included in this study were recruited from a single tertiary hospital. Validation of our findings in other hospital settings and outpatient settings are needed as the findings may differ at non-tertiary hospital settings and are likely to differ in low-tuberculosis burden settings due to differences in population characteristics, empiric treatment decisions and available resources. Second, only a small number of HIV-positive patients were included in the study, precluding our ability to perform a stratified analysis by HIV status. This may be important given that all patients with bacteriologically confirmed tuberculosis and negative chest radiography in our study were HIV positive. Larger studies powered to assess the potential role of chest radiography in HIV-positive and HIV-negative individuals separately are thus needed. Finally, we assessed the role of chest radiography in patients with a negative first-generation Xpert assay. Given the negligible impact, one would predict a similar negative finding among patients with a negative Xpert Ultra result, but this should be confirmed in a prospective study.

In conclusion, despite a strong association between chest radiography findings and presence of active PTB, the addition of chest radiography findings did not improve the performance of clinical parameters traditionally used for empiric tuberculosis treatment decision making in Xpert-negative patients. These findings call for the urgent global implementation of the rapid and highly sensitive Xpert Ultra test in routine clinical practice to improve diagnostic accuracy and early treatment of all tuberculosis cases.

Acknowledgements: The authors would like to thank the staff of the JUMC for their contribution to data collection and laboratory testing, Jimma University Mycobacteriology Research Center staff for their technical assistance and the participants who participated in this study.

Author contributions: W. Kebede, G. Abebe and A. Van Rie designed the study. W. Kebede, G. Abebe, E.K. Gudina and A. Van Rie coordinated the study and the data collection at the site. E. Kedir classified chest radiographic findings. W. Kebede, A. Van Rie and T.N. Tran analysed the data. W. Kebede wrote the first draft. All authors have reviewed the paper and provided comments, and have approved the final version of the manuscript for submission.

Conflict of interest: None declared.

Support statement: This work was supported by the VLIR-UOS network project between Jimma University and a consortium of Flemish Universities, Belgium. The funder had no role in study design, data collection and analysis, decision to publish, or preparation of the manuscript. Funding information for this article has been deposited with the Crossref Funder Registry.

\section{References}

World Health Organization. Global tuberculosis report, 2019. Geneva, WHO, 2019.

2 Auld SC, Shah NS, Cohen T, et al. Where is tuberculosis transmission happening? Insights from the literature, new tools to study transmission and implications for the elimination of tuberculosis. Respirol 2018; 23: 807-817.

3 World Health Organization. Next-generation Xpert MTB/RIF Ultra assay recommended by WHO: Non-inferiority analysis of Xpert MTB/RIF Ultra compared to Xpert MTB/RIF. Geneva, WHO, 2017.

4 World Health Organization. Automated Real-Time Nucleic Acid Amplification Technology for Rapid and Simultaneous Detection of Tuberculosis and Rifampicin Resistance: Xpert MTB/RIF Assay for the Diagnosis of Pulmonary and Extrapulmonary TB in Adults and Children: Policy Update. Geneva, WHO, 2013.

5 World Health Organization. Improving the diagnosis and treatment of smear-negative pulmonary and extrapulmonary tuberculosis among adults and adolescents. Recommendations for HIV-prevalent and resource-constrained settings. Geneva, WHO, 2007.

6 Datiko DG, Guracha EA, Michael E, et al. Sub-national prevalence survey of tuberculosis in rural communities of Ethiopia. BMC Public Health 2019; 19: 295.

7 Kebede AH, Alebachew Z, Tsegaye F, et al. The first population-based national tuberculosis prevalence survey in Ethiopia, 2010-2011. Int J Tuberc Lung Dis 2014; 18: 635-639.

8 Wootton D, Feldman C. The diagnosis of pneumonia requires a chest radiograph (X-ray): yes, no or sometimes? Pneumonia (Nathan) 2014; 5: 1-7.

9 Al Ubaidi BA. The radiological diagnosis of pulmonary tuberculosis (TB) in primary care. J Fam Med Dis Prev 2018; 4: 73.

10 Brady AP. Error and discrepancy in radiology: inevitable or avoidable? Insights Imaging 2017; 8: 171-182.

11 World health organization. Implementing the WHO Stop TB Strategy: A handbook for national TB control programmes. Geneva, WHO, 2008.

12 Jimma University. Jimma University Medical Center Inaugurated Jimma Jimma University. 2018. https://ethio news/2018/12/08/new-medical-center-inaugurated-at-jimma-university/

13 Federal Democratic Republic of Ethiopia Ministry of Health. Guidelines for management of TB, DR-TB and leprosy in Ethiopia. Addis Ababa, Ministry of Health, 2018.

14 Kebede W, Abebe G, Gudina EK, et al. Role of empiric treatment in hospitalized patients with Xpert MTB/ RIF-negative presumptive pulmonary tuberculosis: A prospective cohort study. Int J Infect Dis 2020; 97: 30-37. 
Eisenach K, Demers AM, Jones F. Mycobacteriology Laboratory Sourcebook For Harmonization and Support of Tuberculosis (TB) Clinical Trials. Version 1.0. 2018. pp. 95-103. Available from: https://c-path.org/wp-content/ uploads/2018/06/Mycobacteriology-Laboratory-Sourcebook_Version-1.0_20180307_final2.pdf

16 Bisognin F, Lombardi G, Lombardo D, et al. Improvement of Mycobacterium tuberculosis detection by Xpert MTB/RIF Ultra: A head-to-head comparison on Xpert-negative samples. PLoS One 2018; 13: e0201934.

17 Tuberculosis Coalition for Technical Assistance (TBCTA). HANDBOOK for District Hospitals in Resource Constrained Settings for the Quality Improvement of Chest X-ray Reading in Tuberculosis Suspects. Tuberculosis Coalition for Technical Assistance (TBCTA). The Hague, USAID, 2010.

18 Grobbee DE, Hoes AW. Clinical epidemiology: principles, methods and applications for clinical research Burlington, Jones and Bartlett Learning, 2015; p. 68.

19 World Health Organization. Chest radiography in tuberculosis detection summary of current WHO recommendations and guidance on programmatic approaches. Geneva, WHO, 2016.

20 Zijenah LS. The World Health Organization Recommended TB Diagnostic Tools. In: Tuberculosis. Geneva, IntecOpen, 2018; pp. 71-90.

21 Abebe G, Deribew A, Apers L, et al. Evaluation of the 2007 WHO guideline to diagnose smear negative tuberculosis in an urban hospital in Ethiopia. BMC Infect Dis 2013; 11: 427.

22 Tewolde E, Atnafu A, Kebede T, et al. Evaluating the validity and reliability of chest radiography in the diagnosis of tuberculosis among smear negative pulmonary tuberculosis patients. Ethiop Med J 2015; 53: 83-89.

23 Koole O, Thai S, Khun KE, et al. Evaluation of the 2007 WHO guideline to improve the diagnosis of tuberculosis in ambulatory HIV-positive adults. PLoS One 2011; 6: e18502.

24 Creswell J, Qin ZZ, Gurung R, et al. The performance and yield of tuberculosis testing algorithms using microscopy, chest X-ray, and Xpert MTB/RIF. J Clin Tuberc Other Mycobact Dis 2019; 14: 1-6.

25 World Health Organization. Chest radiography in tuberculosis detection - summary of current WHO recommendations and guidance on programmatic approaches. Geneva, WHO, 2012.

26 Dorman SE, Schumacher SG, Alland D, et al. Xpert MTB/RIF Ultra for detection of Mycobacterium tuberculosis and rifampicin resistance: a prospective multicentre diagnostic accuracy study. Lancet Infect Dis 2018; 18: 76-84.

27 Horne DJ, Kohli M, Zifodya JS, et al. Xpert MTB/RIF and Xpert MTB/RIF Ultra for pulmonary tuberculosis and rifampicin resistance in adults. Cochrane Database Syst Rev 2019; 6: CD009593. 